\title{
Duration Distribution and Up-crossings Rate of Generalized Hyperbolic Processes
}

\author{
Moh'd T. Alodat and Khalid M. Aludaat \\ Department of Statistics, Yarmouk University, Irbid, Jordan
}

\begin{abstract}
A Gaussian process is usually used to model the sea surface elevation in the oceanography. As the depth of the water decreases or the sea severity increases, the sea surface elevation departs from symmetry and Gaussianity. In this paper, a stationary non-Gaussian random process called the generalized hyperbolic process is used as an alternative model. The process generates a family of processes. We derive the rate of up-crossings for this process and the distribution of the height of the process. We also derive the duration distribution of an excursion for the generalized hyperbolic process.

Zusammenfassung: Für gewöhnlich wird ein Gauß Prozess verwendet, um in der Meeresforschung die Höhenangabe der Meeresoberfläche zu modellieren. Wenn die Wassertiefe abnimmt oder der Wellengang zunimmt, dann weicht die Höhe von Symmetrie und Normalverteilung ab. Hier verwenden wir als alternatives Modell einen stationären, nicht-Gauß Prozess, einen so genannten generalisierten hyperbolischen Prozess. Dieser wiederum generiert eine Familie von Prozessen. Wir erhalten für diesen Prozess die Rate der Überschreitungen und die Verteilung der Höhe dieses Prozesses. Wir leiten auch noch für den generalisierten hyperbolischen Prozess die Verteilung der Dauer einer Exkursion ab.
\end{abstract}

Keywords: Gaussian Process, Excursion Set, Duration of an Excursion.

\section{Introduction}

In oceanography, the sea surface elevation, at a fixed location, is modelled by a stationary Gaussian random process. The statistical properties of the sea surface elevation are called the sea state. These properties are very important for the reliability analysis in the ocean engineering (Baxevani et al., 2005). Both the distribution of the maximum sea surface elevation in a given period of time and the distribution of the time spent, above a given level, by the sea surface elevation after an up-crossing (see Figure 1) are of great interest in the oceanography. As the depth of the water decreases or the sea severity increases, the sea surface elevation departs from both symmetry and Gaussianity (Baxevani et al., 2005; Rychlik, 1993; Rychlik and Leadbetter, 2002; Cherneva et al., 2005). Under these sea states, the Gaussian model will not capture the asymmetry in the data. Using the Gaussian model leads to nonconservative estimates. So we should not ignore the asymmetry when modelling the sea (Baxevani et al., 2005). In this case, we need an alternative non-Gaussian stationary random process to model the sea surface elevation. The aim of this paper is to use a non-Gaussian process called the generalized hyperbolic process and to study some of its statistical properties. We derive the rate of up-crossings for this process. We also derive approximations to both the height distribution of the process and the duration distribution of its excursion. 
The following notation is used. We use the symbols $\mathbb{R}$ and $|$.$| for the set of real num-$ bers and for the matrix determinant, respectively. The inverse of a matrix $A$ is denoted by $A^{-1}$. The $d$-dimensional Euclidean space is denoted by $\mathbb{R}^{d}$. We use $\exp (\theta)$ for exponential distribution and $Z \sim N\left(\mu, \sigma^{2}\right)$ to mean that $Z$ is a normal random variable with mean $\mu$ and variance $\sigma^{2}$. The density and cumulative distribution function of the standard normal random variable are $\phi($.$) and \Phi($.$) , respectively.$

Let $Z \sim N\left(\mu, \sigma^{2}\right)$ and $Z^{+}=\max \{0, Z\}$. The following basic result is from Adler (1981) and will be used later

$$
\mathrm{E}\left\{Z^{+}\right\}=\mu(1-\Phi(-\mu / \sigma))+\frac{\sigma}{\sqrt{2 \pi}} \exp \left(-\mu^{2} / 2 \sigma^{2}\right) .
$$

\section{Crossings and Duration of a Process}

Let $X(t), t \in[0, A], A>0$, be a random process. $X(t)$ is said to be differentiable at $t$ in the mean square sense or simply differentiable, if there exists a random process $\dot{X}(t)$ such that

$$
\lim _{h \rightarrow \infty} \mathrm{E}\left\{\left|\frac{X(t+h)-X(t)}{h}-\dot{X}(t)\right|^{2}\right\}=0 .
$$

For a differentiable random process $X(t), t \in[0, A]$, an up-crossing (down-crossing) of the level $u$ occurs at $t_{0} \in[0, A]$, if $X\left(t_{0}\right)=u$ and $\dot{X}\left(t_{0}\right)>0\left(\dot{X}\left(t_{0}\right)<0\right)$, where $\dot{X}(t)$ refers to the derivative of $X(t)$. The length of the interval between an up-crossing and the subsequent down-crossing is called the duration of the excursion of $X(t)$. The excursion set of the process $X(t)$ above a level $u$ is defined to be the set of points $t \in[0, A]$, where $X(t)$ exceeds $u$, i.e., the set $\{t \in[0, A]: X(t) \geq u\}$. This set represents the extreme events of $X(t)$. According to the Poisson Clumping Heuristic ( $\mathrm{PCH})$, the excursion set can be viewed as a set of disjoint independent and identically distributed clumps (Aldous, 1989). So the length of each clump is viewed as a duration. The number of such intervals is approximated a Poisson random variable.

Let $N(u, X)$ be the number of up-crossings of $u$ by $X(t)$. The mean number of the random variable $N(u, X)$ is given (under certain conditions) by the Rice's Formulae

$$
\mathrm{E}\{N(u, X)\}=A \int_{0}^{\infty} y f_{X, \dot{X}}(u, y) d y,
$$

where $f_{X, \dot{X}}(\cdot, \cdot)$ is the joint density of $X$ and $\dot{X}$. The mean value of $N(u, X)$ is very important in many applications of random processes. For example, it can be used to approximate the tail distribution of $\sup _{t \in[0, A]} X(t)$, for large values of $u$ and $A$, i.e.,

$$
\operatorname{Pr}\left\{\sup _{t \in[0, A]} X(t) \geq u\right\} \approx \mathrm{E}\{N(u, X)\} .
$$

For more information about the use of $\mathrm{E}\{N(u, X)\}$ see Leadbetter and Spaniolo (2002) and Rychlik (1993). If $X(t)$ is a differentiable Gaussian process and $\lambda_{2}=\operatorname{var}(\dot{X}(0))$, then

$$
\mathrm{E}\{N(u, X)\}=\frac{A \sqrt{\lambda_{2}}}{2 \pi} \exp \left(-u^{2} / 2\right)
$$




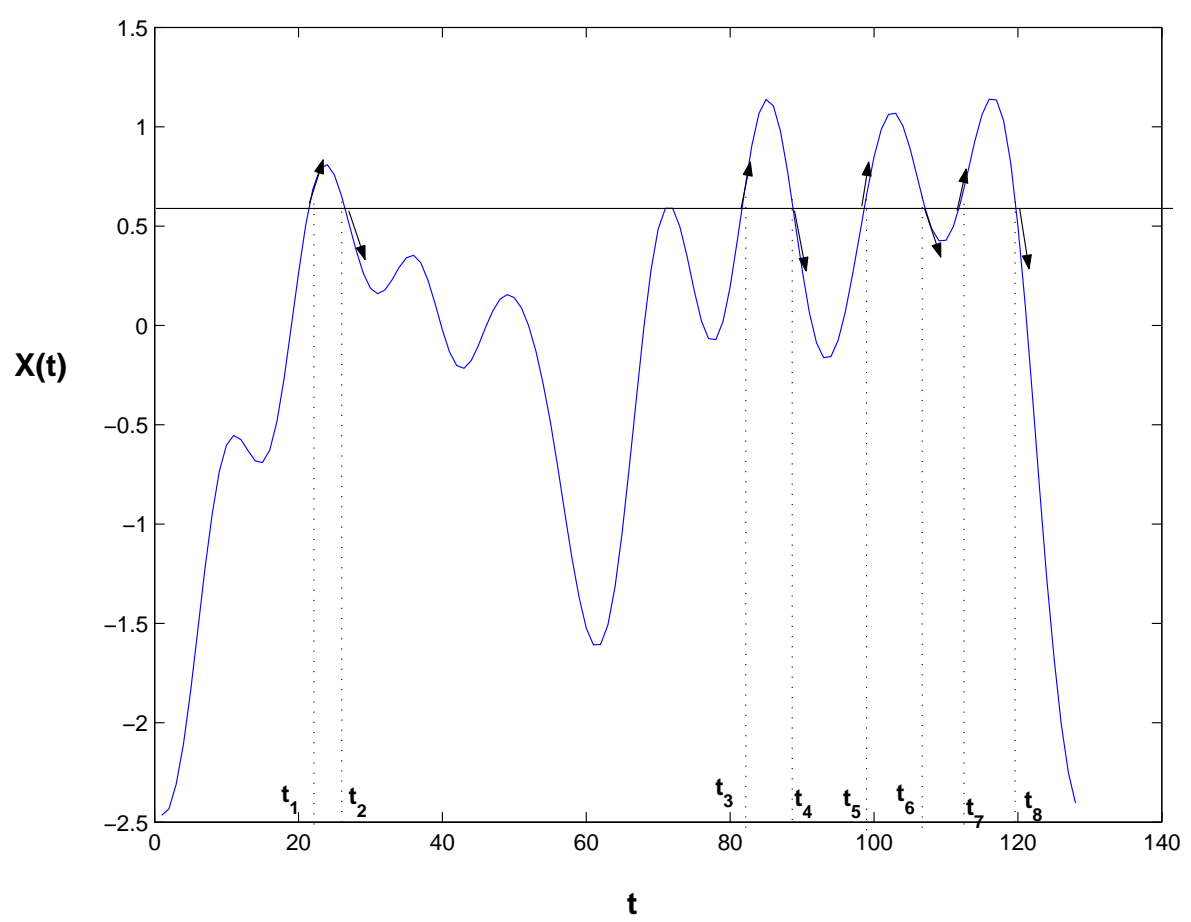

Figure 1: The excursion set of Gaussian process $X(t)$ above the level $u=0.52$ is $\left[t_{1}, t_{2}\right] \cup$ $\left[t_{3}, t_{4}\right] \cup\left[t_{5}, t_{6}\right] \cup\left[t_{7}, t_{8}\right]$. The arrows $\uparrow$ and $\downarrow$ refer to up-crossings and down-crossings, respectively.

\section{Generalized Hyperbolic Distribution}

A random variable $W$ is said to have a generalized inverse Gaussian distribution if it has the density function

$$
f_{W}(w ; \lambda, \chi, \psi)=\frac{\chi^{-\lambda}(\sqrt{\chi \psi})^{\lambda}}{2 K_{\lambda}(\sqrt{\chi \psi})} w^{\lambda-1} \exp \left(-\left(\chi w^{-1}+\psi w\right) / 2\right), \quad w, \chi, \psi>0,
$$

where $K_{\lambda}(\cdot)$ is the modified Bessel function of the third kind with index $\lambda \in \mathbb{R}$. The GIG distribution was introduced by Good (1953) as a model for the species distribution. Jorgensen (1982) studied this distribution in details. We use the notation $W \sim N^{-}(\lambda, \chi, \psi)$ to mean that the random variable $W$ follows a generalized inverse Gaussian distribution with parameters $\lambda, \chi$, and $\psi$. The function $K_{\lambda}(x)$ has the following properties

$$
K_{\lambda}(x)=\left\{\begin{array}{l}
\Gamma(\lambda) 2^{\lambda-1} x^{-\lambda} \quad \text { as } x \downarrow 0, \\
\sqrt{\frac{\pi}{2 x}} \exp (-x) \text { as } x \uparrow \infty,
\end{array}\right.
$$

and

$$
K_{\lambda}(x)=K_{-\lambda}(x)
$$

It can be shown that

$$
\mathrm{E}\left\{W^{\alpha}\right\}=\left(\frac{\chi}{\psi}\right)^{\alpha / 2} \frac{K_{\lambda+\alpha}(\sqrt{\chi \psi})}{K_{\lambda}(\sqrt{\chi \psi})}
$$


Blasild (1981) defines the multivariate generalized hyperbolic distribution as follows: A random vector $V$ is said to have a $d$-dimensional generalized hyperbolic distribution with parameters $\lambda, \psi, \chi, \gamma, \boldsymbol{\mu}$, and $\boldsymbol{\Sigma}$ if it has the joint density

$f_{\mathbf{v}}(\mathbf{v})=c \frac{K_{\lambda-\frac{d}{2}}\left(\sqrt{\left(\chi+(\mathbf{v}-\boldsymbol{\mu})^{T} \boldsymbol{\Sigma}^{-1}(\mathbf{v}-\boldsymbol{\mu})\right)\left(\psi+\gamma^{T} \boldsymbol{\Sigma}^{-1} \boldsymbol{\gamma}\right)}\right) \exp \left((\mathbf{v}-\boldsymbol{\mu})^{T} \boldsymbol{\Sigma}^{-1} \gamma\right)}{\left(\sqrt{\left(\psi+(\mathbf{v}-\boldsymbol{\mu})^{T} \boldsymbol{\Sigma}^{-1}(\mathbf{v}-\boldsymbol{\mu})\right)\left(\psi+\gamma^{T} \boldsymbol{\Sigma}^{-1} \gamma\right)}\right)^{\frac{d}{2}-\lambda}}$

where $\mathbf{v}, \boldsymbol{\gamma}, \boldsymbol{\mu} \in \mathbb{R}^{d}, \boldsymbol{\Sigma}$ is a $d \times d$ matrix, and

$$
c=\frac{(\sqrt{\chi \psi})^{-\lambda} \psi^{\lambda}\left(\psi+\gamma^{T} \boldsymbol{\Sigma}^{-1} \gamma\right)^{\frac{d}{2}-\lambda}}{(2 \pi)^{\frac{d}{2}}|\Sigma|^{\frac{1}{2}} K_{\lambda}(\sqrt{\chi \psi})} .
$$

Any $d$-dimensional generalized hyperbolic distribution has the following stochastic representation

$$
\mathbf{V}=\boldsymbol{\mu}+\gamma W+\sqrt{W} \mathbf{Z},
$$

where $\boldsymbol{\mu}, \boldsymbol{\gamma}$ are constant vectors and $W$ is a GIG random variable independent of $\mathbf{Z}$, a multivariate normal vector with mean vector $\mathbf{0}$ and covariance matrix $\boldsymbol{\Sigma}$. The mean vector of $\mathbf{V}$ is given by

$$
\mathrm{E}\{\mathbf{V}\}=\boldsymbol{\mu}+\boldsymbol{\gamma}\left(\frac{\chi}{\psi}\right)^{1 / 2} \frac{K_{\lambda+1}(\sqrt{\chi \psi})}{K_{\lambda}(\sqrt{\chi \psi})}
$$

Consider the following partitions of $\mathbf{V}, \boldsymbol{\gamma}, \boldsymbol{\mu}$, and $\Sigma$ :

$$
\mathbf{V}=\left(\begin{array}{l}
\boldsymbol{V}_{1} \\
\boldsymbol{V}_{2}
\end{array}\right), \quad \boldsymbol{\gamma}=\left(\begin{array}{l}
\gamma_{1} \\
\gamma_{2}
\end{array}\right), \quad \boldsymbol{\mu}=\left(\begin{array}{l}
\boldsymbol{\mu}_{1} \\
\boldsymbol{\mu}_{2}
\end{array}\right), \quad \boldsymbol{\Sigma}=\left(\begin{array}{ll}
\boldsymbol{\Sigma}_{11} & \boldsymbol{\Sigma}_{12} \\
\boldsymbol{\Sigma}_{21} & \boldsymbol{\Sigma}_{22}
\end{array}\right)
$$

Then the conditional distribution of $\boldsymbol{V}_{2}$ given $\boldsymbol{V}_{1}=\boldsymbol{v}_{1}$ is multivariate generalized hyperbolic distribution with parameters

$$
\begin{aligned}
& \tilde{\lambda}=\lambda-\frac{k_{1}}{2}, \quad \tilde{\psi}=\psi+\gamma_{1}^{T} \Sigma_{11}^{-1} \gamma_{1}, \\
& \tilde{\chi}=\chi+\left(\boldsymbol{v}_{1}-\boldsymbol{\mu}_{1}\right)^{T} \boldsymbol{\Sigma}_{11}^{-1}\left(\boldsymbol{v}_{1}-\boldsymbol{\mu}_{1}\right), \quad \tilde{\boldsymbol{\gamma}}=\boldsymbol{\gamma}_{2}-\boldsymbol{\gamma}_{1}^{T} \boldsymbol{\Sigma}_{11}^{-1} \boldsymbol{\Sigma}_{12}, \\
& \tilde{\boldsymbol{\mu}}=\boldsymbol{\mu}_{2}+\left(\boldsymbol{v}_{1}-\boldsymbol{\mu}_{1}\right)^{T} \boldsymbol{\Sigma}_{11}^{-1} \boldsymbol{\Sigma}_{12}, \quad \tilde{\boldsymbol{\Sigma}}=\boldsymbol{\Sigma}_{22}-\boldsymbol{\Sigma}_{21} \boldsymbol{\Sigma}_{11}^{-1} \boldsymbol{\Sigma}_{12} .
\end{aligned}
$$

The one-dimensional generalized hyperbolic distribution was introduced by BarndorffNielsen (1977). In the recent years, the generalized hyperbolic distributions were used by many authors to fit the financial time series by stochastic processes (Rydberg, 1997; Bibby and Sorensen, 1997; Raible, 2000; Schoutens, 2003; Cont and Tankov, 2004; Emmer and Klüppelberg, 2004). For example, if $S_{t}, t=1,2, \ldots$, represents the sequence of prices of a stock, then the sequence of the $\log$ returns, $X_{t}=\log \left(S_{t}\right)-\log \left(S_{t-1}\right), t=1,2, \ldots$, is well fitted by a generalized hyperbolic process.

\section{Generalized Hyperbolic Process}

In this section, we define a continuous version of the generalized hyperbolic process. Let $X(t), t \in[0, A]$, be a stationary and differentiable Gaussian random process with mean 
zero and variance equal one. We define the generalized hyperbolic process $Y(t)$ by

$$
Y(t)=\mu+\gamma W+\sqrt{W} X(t)
$$

where $\gamma, \mu \in \mathbb{R}$ and $W \sim N^{-}(\lambda, \chi, \psi)$. It can be noted that every finite dimensional distribution of the process $Y(t)$ is a multivariate generalized hyperbolic distribution.

It easy to see that stationarity of $X(t)$ implies the stationarity of $Y(t)$. If $\mathbf{R}_{X}(t)$ is the covariance function of $X(t)$, then the covariance function of $Y(t)$ is

$$
\begin{aligned}
\mathbf{R}_{Y}(t) & =\mathrm{E}\{Y(t) Y(0)\}, \\
& =\mathrm{E}\{(\mu+\gamma W+\sqrt{W} X(t))(\mu+\gamma W+\sqrt{W} X(0))\}, \\
& =\gamma^{2} \operatorname{var}(W)+\mathrm{E}\{W\} \mathbf{R}_{X}(t) .
\end{aligned}
$$

The last equation implies that $Y(t)$ is differentiable in the mean square sense and $\dot{Y}(t)=$ $\sqrt{W} \dot{X}(t)$. The random variables $X(0)$ and $X \dot{(0)}$ are independent and normally distributed such that $X=X(0) \sim N(0,1)$ and $\dot{X}=\dot{X}(0) \sim N\left(0, \lambda_{2}\right)$. Conditional on $W$, the joint distribution of $(Y, \dot{Y})=(Y(0), \dot{Y}(0))$ is bivariate normal with joint density function

$$
f_{Y, \dot{Y}}(y, \dot{y} \mid W=w)=\frac{1}{w \sqrt{\lambda_{2}}} \phi\left(\frac{y-\mu-\gamma \mathrm{E}\{W\}}{\sqrt{w}}\right) \phi\left(\frac{\dot{y}}{\sqrt{w \lambda_{2}}}\right),
$$

with $\mathrm{E}\{W\}$ given by (4). Using total probability law and the Fubini's theorem, we get

$$
\begin{aligned}
\mathrm{E}\{N(u, Y)\} & =A \int_{0}^{\infty} \dot{y} f_{Y, \dot{Y}}(u, \dot{y}) d \dot{y} \\
& =A \int_{0}^{\infty} \int_{0}^{\infty} \dot{y} f_{Y, \dot{Y}}(u, \dot{y} \mid W=w) f_{W}(w) d w d \dot{y} \\
& =A \int_{0}^{\infty} \int_{0}^{\infty} \frac{\dot{y}}{w \sqrt{\lambda_{2}}} \phi\left(\frac{u-\mu-\gamma \mathrm{E}\{W\}}{\sqrt{w}}\right) \phi\left(\frac{\dot{y}}{\sqrt{w \lambda_{2}}}\right) f_{W}(w) d \dot{y} d w \\
& =A \int_{0}^{\infty} \frac{1}{\sqrt{w}} \phi\left(\frac{u-\mu-\gamma \mathrm{E}\{W\}}{\sqrt{w}}\right) \mathrm{E}\left\{\dot{Y}^{+} \mid W=w\right\} f_{W}(w) d w \\
& =A \int_{0}^{\infty} \frac{1}{\sqrt{w}} \phi\left(\frac{u-\mu-\gamma \mathrm{E}\{W\}}{\sqrt{w}}\right)\left(\sqrt{\frac{w \lambda_{2}}{2 \pi}}\right) f_{W}(w) d w \\
& =\frac{A \sqrt{\lambda_{2}}}{2 \pi} \frac{\chi^{-\lambda}(\sqrt{\chi \psi})^{\lambda}}{2 K_{\lambda}(\sqrt{\chi \psi})} \int_{0}^{\infty} w^{\lambda-1} \exp \left(-\frac{1}{2}\left(\frac{\chi^{*}}{w}+\psi w\right)\right) d w
\end{aligned}
$$

where $\chi^{*}=(u-\mu-\gamma \mathrm{E}\{W\})^{2}+\chi$. Using (1), we simplify the last equation to

$$
\mathrm{E}\{N(u, Y)\}=\frac{A \sqrt{\lambda_{2}}}{2 \pi}\left(\frac{\chi^{*}}{\chi}\right)^{\lambda / 2} \frac{K_{\lambda}\left(\sqrt{\chi^{*} \psi}\right)}{K_{\lambda}(\sqrt{\chi \psi})} .
$$

According to the $\mathrm{PCH}$, the number of up-crossings, $N(u, Y)$, can be approximated by a Poisson point process with mean $\mathrm{E}\{N(u, Y)\}$. We may use the standard parametric methods for the Poisson distribution to estimate the parameters $\lambda, \psi$, and $\chi$ if realizations of the point process $N(u, X)$ are available. 


\section{Special Cases}

If $S$ is a chi-square random variable with $\nu$ degrees of freedom, then the random variable $W=\nu / S$ has density

$$
f_{W}(w)=\frac{w^{-\nu / 2-1} \exp (-\nu / 2 w)}{\Gamma(\nu / 2) 2^{\nu / 2} \nu^{\nu / 2}}, \quad w>0
$$

which is $N^{-}(-\nu / 2, \nu, \psi)$ as $\psi \rightarrow 0$. For the case $\mu=\gamma=0, Y(t)$ is a Student- $t$ random variable with $\nu$ degrees of freedom. Therefore,

$$
\mathrm{E}\{N(u, Y)\}=\frac{A \sqrt{\lambda_{2}}}{2 \pi}\left(\frac{u^{2}+\nu}{\nu}\right)^{-\nu / 4} \frac{K_{-\nu / 2}\left(\sqrt{\chi^{*} \psi}\right)}{K_{-\nu / 2}(\sqrt{\chi \psi})} .
$$

Using (2) and (3), it is easy to show that

$$
\lim _{\psi \rightarrow 0} \frac{K_{-\nu / 2}\left(\sqrt{\chi^{*} \psi}\right)}{K_{-\nu / 2}(\sqrt{\chi \psi})}=\left(1+\frac{u^{2}}{\nu}\right)^{-\nu / 4} .
$$

This leads to

$$
\mathrm{E}\{N(u, Y)\}=\frac{A \sqrt{\lambda_{2}}}{2 \pi}\left(1+\frac{u^{2}}{\nu}\right)^{-\nu / 2}
$$

which is the rate of the up-crossings of the Student- $t$ process given in Alodat and Aludaat (2006). As $\nu \rightarrow \infty$, the Student distribution goes to the Gaussian distribution. Note, as $\nu \rightarrow \infty$, the right hand side of (8) tends to $\mathrm{E}\{N(u, X)\}$, the expected number of up-crossings of $u$ by the Gaussian process $X(t)$.

\section{Height Distribution of $Y(t)$}

The distribution of the height of $Y(t)$ above the level $u$ is defined to be the distribution of $Y(0)-u$, given that $Y(t)$ has a local maximum of height exceeds $u$ at $t=0$. This can be calculated according to Rychlik (1993). Since the expected number of up-crossings of $Y(t)$ approximates the expected number of local maxima of $Y(t)$, we may calculate the height distribution of $Y(t)$ according to Adler (1981, p. 158). So as $u \rightarrow \infty$, the ratio $\mathrm{E}\{N(u+y, Y)\} / \mathrm{E}\{N(u, Y)\}$ tends to $1-G(y)$, where $G(y)$ is the height distribution of the process $Y(t)$. Using (2) and taking the limit as $u \rightarrow \infty$, we get

$$
\begin{aligned}
1-G(y)=\lim _{u \rightarrow \infty} \exp ( & -\sqrt{\left((u+y-\mu-\gamma \mathrm{E}\{W\})^{2}+\chi\right) \psi} \\
& \left.+\sqrt{\left((u-\mu-\gamma \mathrm{E}\{W\})^{2}+\chi\right) \psi}\right) \\
= & \exp (-\sqrt{\psi} y), \quad y, \psi>0
\end{aligned}
$$




\section{Approximating the Duration Distribution}

In this section, we suggest an approximation to the duration distribution of $Y(t)$. To do this we propose the following steps:

1. Approximate $Y(t)$ near its local maximum at $t=0$ by $\tilde{Y}(t)=Y(0)+\dot{Y}(0) t+\frac{1}{2} \ddot{Y} t^{2}$, the Taylor polynomial of degree 2 .

2. Replace the random variable $\ddot{Y}$ by $\mathrm{E}\{\ddot{Y} \mid Y=u, \dot{Y}=0\}$.

3. The difference between the two real roots of the equation $u=\tilde{Y}(t)$ represents an approximation to the duration of $Y(t)$.

4. Find the distribution of the random variable in step 3.

We go over this step by step:

Step 1. Since $Y(t)$ has a local maximum at $t=0$, the Taylor polynomial of degree 2 is reduced to $\tilde{Y}(t)=Y(0)+\frac{1}{2} \ddot{Y} t^{2}$.

Step 2. Note that $\dot{Y}(t)=\sqrt{W} \dot{X}(t)$ and $\ddot{Y}(t)=\sqrt{W} \ddot{X}(t)$. Using (5), we see that the joint distribution of $V=(Y, \dot{Y}, \ddot{Y})=(Y(0), \dot{Y}(0), \ddot{Y}(0))$ is multivariate generalized hyperbolic distribution with parameters $\lambda, \psi, \chi$

$$
\gamma^{*}=\left(\begin{array}{l}
\gamma \\
0 \\
0
\end{array}\right), \quad \boldsymbol{\mu}=\left(\begin{array}{l}
\mu \\
0 \\
0
\end{array}\right), \quad \boldsymbol{\Sigma}=\operatorname{cov}(X, \dot{X}, \ddot{X})=\left(\begin{array}{ccc}
1 & 0 & -\lambda_{2} \\
0 & \lambda_{2} & 0 \\
-\lambda_{2} & 0 & \lambda_{2}^{*}
\end{array}\right)
$$

where $\lambda_{2}^{*}=\operatorname{var}(\ddot{X})$. The conditional distribution of $\ddot{Y}$, given $Y=u$ and $\dot{Y}=0$, is univariate generalized hyperbolic distribution with parameters computed from the equations (??)-(??) as follows

$$
\gamma_{1}=\left(\begin{array}{l}
\gamma \\
0
\end{array}\right), \gamma_{2}=\mathbf{0}, \boldsymbol{\mu}_{1}=\left(\begin{array}{l}
\mu \\
0
\end{array}\right), \boldsymbol{\mu}_{2}=0, \boldsymbol{\Sigma}_{11}=\left(\begin{array}{cc}
1 & 0 \\
0 & \lambda_{2}
\end{array}\right), \boldsymbol{\Sigma}_{12}=\left(\begin{array}{c}
-\lambda_{2} \\
0
\end{array}\right), \boldsymbol{\Sigma}_{22}=\lambda_{2}^{*} .
$$

This gives $\tilde{\lambda}=\lambda-1, \tilde{\psi}=\psi+\gamma^{2}, \tilde{\chi}=\chi+(u-\mu)^{2}, \tilde{\gamma}=\gamma \lambda_{2}, \tilde{\mu}=-\lambda_{2}(u-\mu)$, and $\tilde{\Sigma}=\lambda^{*}-\lambda_{2}^{2}$. Using (6) we get

$$
\begin{aligned}
\mathrm{E}\{\ddot{Y} \mid Y=u, \dot{Y}=0\} & =\tilde{\mu}+\tilde{\gamma}\left(\frac{\tilde{\chi}}{\tilde{\psi}}\right)^{1 / 2} \frac{K_{\tilde{\lambda}+1}(\sqrt{\tilde{\chi} \tilde{\psi}})}{K_{\tilde{\lambda}}(\sqrt{\tilde{\chi} \tilde{\psi}})} \\
& =-\lambda_{2}(u-\mu)+\gamma \lambda_{2} \frac{\sqrt{\chi+(u-\mu)^{2}}}{\sqrt{\psi+\gamma^{2}}} \frac{K_{\lambda}\left(\sqrt{\left(\psi+\gamma^{2}\right)\left(\chi+(u-\mu)^{2}\right)}\right)}{K_{\lambda-1}\left(\sqrt{\left(\psi+\gamma^{2}\right)\left(\chi+(u-\mu)^{2}\right)}\right)} .
\end{aligned}
$$

Using (2), we get

$$
\begin{aligned}
\mathrm{E}\{\ddot{Y} \mid Y=u, \dot{Y}=0\} & \approx-\lambda_{2}(u-\mu)\left(1-\gamma \frac{\sqrt{\frac{\chi}{(u-\mu)^{2}}+1}}{\sqrt{\psi+\gamma^{2}}}\right) \\
& =-\lambda(u-\mu) h(\gamma, \psi, \mu, \gamma, u), \text { say }
\end{aligned}
$$



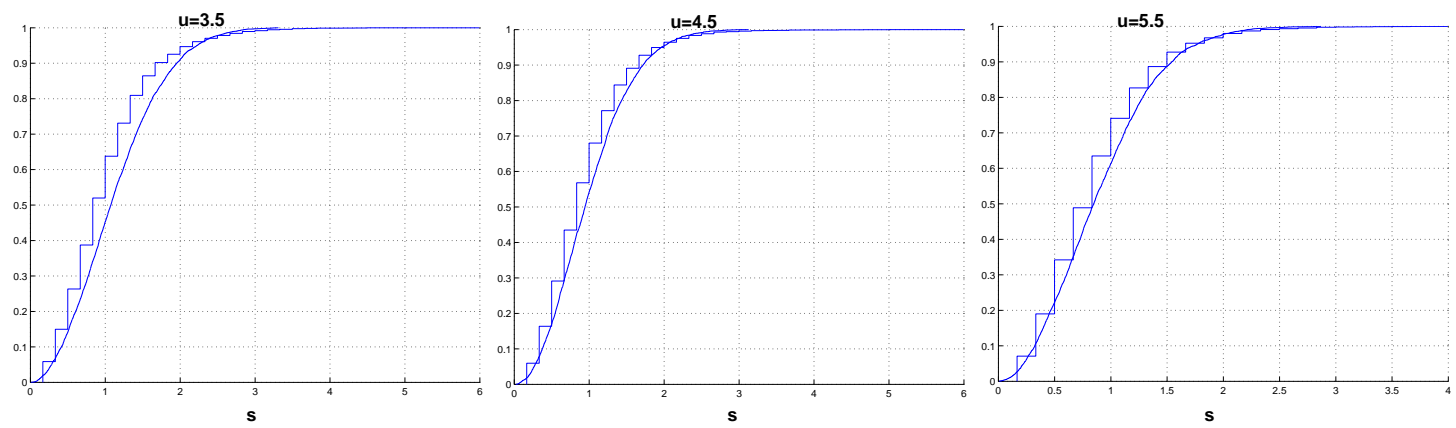

Figure 2: Empirical distributions for large simulated samples from the two distributions of $S$; exact (smooth) and approximate (steps).

for large $u$. If we set $h=h(\gamma, \psi, \mu, \gamma, u)$, then $\tilde{Y}(t)=Y(0)-\frac{1}{2} \lambda_{2}(u-\mu) h t^{2}$.

Step 3. The roots of the last equation are $t=\mp \sqrt{2(Y-u) / \lambda(u-\mu) h}$. The random variable $S=2 \sqrt{2(Y-u) / \lambda(u-\mu) h}$ approximates the duration of $Y(t)$ above the level $u$. Section 6 provides the distribution of the random variable $Y-u$ which is $\exp (1 / \sqrt{\psi})$. So the density function of $S$ is given by

$$
f_{S}(s)=\frac{1}{4} \lambda \sqrt{\psi} h(u-\mu) s \exp \left(-\frac{1}{8} \lambda \sqrt{\psi} h(u-\mu)^{2} s^{2}\right), \quad \text { for } s>0,
$$

and $f_{S}(s)=0$ for $s<0$.

\section{Simulation of $Y(t)$}

To simulate from the generalized hyperbolic process $Y(t)$ we follow the following steps

1. Simulate a generalized inverse Gaussian variable $W$.

2. Simulate a Gaussian process $X(t)$.

3. Realize $Y(t)$ as $Y(t)=\mu+\gamma W+\sqrt{W} X(t)$.

Atkinson (1982) gives an algorithm to simulate from a generalized inverse Gaussian distribution. We follow the above algorithm to simulate a large sample from the exact duration distribution of the generalized hyperbolic process for the case $\mu=0, \gamma=0$ and $W \sim \exp (1)$. We also simulated a large sample from the density (9). The two samples are described by their empirical distribution functions in Figure 2. It can be noted that the maximum difference between the two empirical distribution functions is very small for large values of $u$, which means that the approximation is good.

\section{Conclusions}

In this paper, we used a non-Gaussian process called the generalized hyperbolic process. This process generates a family of random processes. As limit processes, the Gaussian 
and the Student processes are members of this family. This makes the generalized hyperbolic process a flexible model in many practical problems. We derived a closed form approximation to the duration distribution its excursion. Simulation shows that the approximation works well.

\section{Acknowledgements}

The authors thank the two referees for their comments which helped us to improve the paper.

\section{References}

Adler, R. (1981). The Geometry of Random Fields. New York: John Wiley and Sons.

Aldous, D. (1989). Probability Approximations via the Poisson Clumping Heuristic. New York: Springer-Verlag.

Alodat, M. T., and Aludaat, K. M. (2006). Testing of statistical hypothesis using the student process. (Submitted for publication)

Atkinson, A. C. (1982). The simulation of generalized inverse Gaussian and hyperbolic random variables. SIAM Journal of Scientific and Statsitical Computing, 3, 502515.

Barndorff-Nielsen, O. E. (1977). Exponentially decreasing distributions for the logarithm of particle size. In Proceedings of the royal society of london a (Vol. 353, p. 401419).

Baxevani, A., Hagberg, O., and Rychlik, I. (2005). Note on the distribution of extreme wave crests. In Proceeding of OMAE2005 24th International Conference on Offshore Mechanics and Arctic Engineering.

Bibby, B. M., and Sorensen, M. (1997). A hyperbolic diffusion model for stock prices. Finance and Stochastics, 1, 25-41.

Blasild, P. (1981). The two-dimensional hyperbolic distribution and related distributions, with applications to Johannsen's bean data. Biometrika, 68, 251-263.

Cherneva, Z., Petrova, P., Andreeva, N., and Soares, C. G. (2005). Probability distribution of peaks, troughs and heights of wind waves measured in the black sea coastal zone. Costal Engineering, 52, 599-615.

Cont, R., and Tankov, P. (2004). Financial Modeling with Jump Processes. London: Chapman and Hall/CRC.

Emmer, S., and Klüppelberg, C. (2004). Optimal portfolios when stock prices follow an exponential Lévy process. Finance and Stochastics, 8, 17-44.

Good, I. J. (1953). The population frequencies of species and the etimation of population parameters. Biometrika, 40, 237-260.

Jorgensen, B. (1982). Statistical Properties of the Generalized Inverse Gaussian Distribution. New York: Springer-Verlag.

Leadbetter, M. R., and Spaniolo, G. V. (2002). On statistics at level crossings by a stationary process. Statistica Neerlandica, 56, 152-164. 
Raible, S. (2000). Lévy Processes in Finance: Theory, Numerics and Empirical Facts. Unpublished doctoral dissertation, Mathematische Fakultat, AlbertLudwigs-Universitat Freiburg im Breisgau.

Rychlik, I. (1993). On the narrow-band approximation for expected fatigue damage. Probabilistic Engineering Mechanics, 8, 1-4.

Rychlik, I., and Leadbetter, M. R. (2002). Analysis of ocean waves by crossings and osciallation intensities. International Journal of Offshore and Polar Engineering, $10,282-289$.

Rydberg, T. H. (1997). The normal inverse Gaussian Lévy process: Simulation and approximation. Communications in Statistics: Stochastics Models, 13, 887-910.

Schoutens, W. (2003). Lévy Process in Finance-Pricing Financial Derivatives. Chichester: John Wiley and Sons.

Authors' address:

Moh'd T. Alodat and Khalid M. Aludaat

Department of Statistics

Yarmouk University

Irbid, Jordan

E-mails: malodat@yu.edu.jo and aludaatkm@hotmail.com 